'Departamento de Nutrición,

Diabetes y Metabolismo,

Escuela de Medicina, Pontificia Universidad Católica de Chile. Santiago, Chile.

Instituto de Investigación en Ciencias Odontológicas, Facultad de Odontología, Universidad de

Chile. Santiago, Chile.

${ }^{3}$ Instituto de Nutrición y

Tecnología de los Alimentos

(INTA), Universidad de Chile. Santiago, Chile.

${ }^{4}$ Departamento de Pediatría y Cirugía Infantil. Universidad de la

Frontera. Temuco, Chile.

aTecnólogo Médico, Doctor

(Ph.D.) en Ciencias Biomédicas.

bBióloga, Magister en Ciencias.

'Biólogo, Doctor (Ph.D.) en

Ciencias Biológicas.

Fuente de Financiamiento: Proyecto FONDECYT 1090388

Recibido el 27 de noviembre de 2013, aceptado el 13 de marzo de 2014

Correspondencia: Prof. José Luis Santos Alameda 340, Edificio de Gastroenterología $4^{\text {to }}$ piso. Santiago, Chile. Telefono: +56-2-3543862 Fax: +56-2-6338298 jsantos@med.puc.cl

\section{Polimorfismos genéticos de interleuquina 6 (IL6), IL6R e IL18: asociación con componentes del síndrome metabólico en niños chilenos con obesidad}

\author{
JOSÉ SUAZO ${ }^{1,2, a}$, SUSAN VALERIE SMALLEY ${ }^{1, b}$, \\ MARÍA ISABEL HODGSON ${ }^{1}$, GERARDO WEISSTAUB ${ }^{3}$, \\ ANDREA GONZÁLEZ ${ }^{1,4}$, JOSÉ LUIS SANTOS ${ }^{1, c}$
}

\section{Association between genetic polymorphisms of interleukin 6 (IL6), IL6R and IL18 with metabolic syndrome in obese Chilean children}

Background: Metabolic Syndrome (MS) is highly prevalent among obese children and adolescents and is considered a predictor for the development of type 2 diabetes mellitus and cardiovascular disease. Obesity is associated with an increase in circulating levels of interleukins 6 (IL6) and 18 (IL18), which in turn would depend on polymorphisms of IL6, IL6R and IL18 genes. Aim: To evaluate the association between genetic polymorphisms of IL6 ( $r$ 1800795, rs 1800796 and rs1800797), IL6R (rs2228145) and IL18 (rs360719, rs187238 and rs204355) and MS and/or its components in a sample of Chilean obese children. Patients and Methods: These polymorphisms were genotyped in 259 obese children aged 10 \pm 2 years with a body mass index of $26.1 \pm 4.1 \mathrm{~kg} / \mathrm{m}^{2}$. Sixty eight had metabolic syndrome (26.3\%). The association of their alleles, genotypes and haplotypes with the MS and its components was assessed. Results: IL6, IL6R and IL18 variants showed no association with SM nor with any of the phenotypes that compose it. However, IL18 haplotypes ( $r$ 360719-rs187238-rs204355) TCT and CGT were associated with triglycerides $\leq 110 \mathrm{mg} / \mathrm{dL}$ and $H D L<40 \mathrm{mg} / \mathrm{dL}$, respectively. Conclusions: IL6 and IL6R variants are not associated with MS or with any of its phenotypes. Although an association between IL18 haplotypes and certain MS component has been detected herein, it is necessary to replicate our findings in independent studies due to the low frequency of these allele combinations detected in our sample.

(Rev Med Chile 2014; 142: 290-298)

Key words: Haplotypes; Interleukin-18; Metabolic syndrome X.
4 1 síndrome metabólico (SM) se define por la combinación de obesidad central, hipertensión arterial, dislipidemia e hiperglicemia. Si bien el significado biológico del SM es controversial ${ }^{1}$, este es considerado como un factor predictor del desarrollo patologías como diabetes mellitus tipo 2 (DM2) y enfermedad cardiovascular, ambas condiciones relacionadas con la resistencia a la insulina $(\mathrm{RI})^{2}$. Países desarrollados y en vías de desarrollo registran un incremento en la prevalencia de obesidad en niños y adolescentes ( $14 \%$ en 2000 a $\sim 17 \%$ en 
$2010)^{3}$. En Chile, la rápida transición epidemiológica y nutricional ha triplicado la prevalencia de obesidad infantil en las últimas dos décadas ${ }^{4,5}$. La relación entre sobrepeso/obesidad infantil y RI ha enfocado la investigación en el SM como predictor de enfermedades de la vida adulta ${ }^{6}$. La prevalencia global de SM en niños y adolescentes se ha estimado en $4,2 \%$ y $8,6 \%$, respectivamente ${ }^{7,8}$. Sin embargo, estas tasas se incrementan considerablemente cuando sólo se consideran niños obesos $(19-28 \%)^{7-9}$. Recientemente, nuestro grupo ha reportado una prevalencia de $26,3 \%$ en una población de niñas y niños obesos (6-12 años) de la ciudad de Santiago, Chile ${ }^{10}$.

La agregación familiar del SM y las diferencias en su prevalencia de acuerdo al origen étnico, ha permitido postular la existencia de factores genéticos en su expresión ${ }^{7,8,11,12}$. Estudios de asociación de genoma completo han relacionado factores genéticos con cada uno de los componentes del $\mathrm{SM}^{13}$. Usando herramientas bioinformáticas, Sookoian y Pirola ${ }^{14}$, han identificado una serie de genes y rutas biológicas comunes asociadas al riesgo de SM, muchos de ellos relacionados con la respuesta inmune e inflamatoria.

Las interleuquinas (IL) incluyen una serie de péptidos que ejercen múltiples efectos biológicos, y que han sido estudiadas tradicionalmente desde la perspectiva de su relación con procesos inflamatorios ${ }^{15}$. La acumulación de grasa corporal propia de la obesidad se acompaña de un estado inflamatorio de baja intensidad que se caracteriza por hipertrofia e hiperplasia del tejido adiposo, reclutamiento de macrófagos, y producción incrementada de adipoquinas tales como IL6, TNF- $\alpha$, IL-1 $\beta$ y leptina, procesos que podrían activarse durante la infancia ${ }^{16}$. A estas moléculas podemos agregar el efecto de IL18 sobre la modulación de la ingesta y la acumulación de adiposidad demostrada en ratones deficientes del gen $\mathrm{Il}_{1} 8^{17}$. Dada su relación con estados inflamatorios, los niveles de IL6, de su receptor soluble (IL6sR) y de interleuquina 18 (IL18) han sido correlacionados con obesidad, RI y DM2 ${ }^{18-23}$. Por lo tanto, es posible postular que los niveles de IL6, IL6sR e IL18 pueden estar relacionados con SM o alguno de sus componentes.

Existen evidencias para postular que los niveles circulantes de IL6 e IL6sR estarían influenciados por polimorfismos genéticos ${ }^{24-28}$. Se ha demostrado que los polimorfismos de base simple (SNPs) en la región promotora del gen IL6 (7p21), rs1800795 (-174G > C), rs1800796 (-572 $\mathrm{G}>\mathrm{C})$ y rs 1800797 (-598G $>$ C), ejercen un rol conjunto en la actividad transcripcional de dicho promotor $^{29}$. El gen IL6R (1q21.3), codifica tanto para un receptor asociado a membrana como para IL6sR, como producto de un splicing alternativo y/o de un procesamiento proteico ${ }^{30}$. La variante no sinónima rs2228145 (p.Asp358Ala) en IL6R parece alterar la estructura proteica extracelular del receptor asociado a membrana, estimulando así su procesamiento hacia la producción de su forma soluble ${ }^{31}$. IL6sR es necesario para ejercer el efecto de IL6 en células que no expresan dicho receptor $^{32}$. Por su parte, los polimorfismos de la región promotora del gen IL18 (11q23.1), rs360719 $(-1297 \mathrm{~T}>\mathrm{C})$ y rs $187238(-137 \mathrm{G}>\mathrm{C})$ y la variante intrónica rs204355 $(-5848 \mathrm{~T}>\mathrm{C})$ han demostrado tener influencia sobre los niveles circulantes de IL18 ${ }^{33-35}$. Además, estos polimorfismos de IL6, IL6R e IL18 han sido asociados previamente tanto con SM como con sus componentes, en poblaciones de diferente origen étnico ${ }^{21,30,37-40}$. En el caso de la población chilena, se reporta un estudio que asocia a rs2228145 con el índice de masa corporal (IMC) en mujeres con DM1 ${ }^{41}$.

Dados los antecedentes antes expuestos, el objetivo de este estudio es replicar la asociación, a nivel alélico, genotípico y haplotípico, existente entre polimorfismos genéticos de IL6 (rs1800795, rs1800796 y rs1800797), de IL6R (rs2228145) y de IL18 (rs360719, rs187238 y rs204355) con el SM y/o sus componentes. Para ello, hemos considerado una muestra de niñas y niños con obesidad en la que se ha descrito previamente tanto su perfil metabólico como la prevalencia de $\mathrm{SM}^{10}$.

\section{Sujetos y Métodos}

\section{Participantes}

En un estudio transversal, se reclutaron 259 niños y niñas chilenas ( 122 niñas y 137 niños; edad 6-12 años) con IMC $\geq$ percentil 95 de acuerdo a $\mathrm{NCHS} / \mathrm{CDC}^{42}$. El perfil antropométrico, metabólico y del estado de madurez puberal de esta muestra se describe en la Tabla 1 y en una publicación anterior de nuestro grupo ${ }^{10}$. Dicho artículo reporta un IMC Z-score promedio de 2,12 (mínimo 1,30; máximo 3,33). Respecto al estadio puberal, esta muestra presenta la siguiente distribución: $61,8 \%$ Tanner 1; 22,9\% Tanner 2; 11,6\% Tanner 3; 2,8\% 
Tabla 1. Características antropométricas y metabólicas de 259 niñas y niños chilenos con obesidad

\begin{tabular}{|c|c|c|c|c|}
\hline & $\begin{array}{c}\text { Total muestra } \\
(n=259) \\
\text { Promedio } \pm \text { DS }\end{array}$ & $\begin{array}{c}S^{\mathrm{a}} \\
(n=68) \\
\text { Promedio } \pm \mathrm{DS}\end{array}$ & $\begin{array}{c}\text { No-SM }^{b} \\
(n=191) \\
\text { Promedio } \pm \text { DS }\end{array}$ & $\mathbf{p}^{c}$ \\
\hline Edad (años) & $9,6 \pm 1,8$ & $10,1 \pm 1,7$ & $9,4 \pm 1,8$ & 0,014 \\
\hline Peso (kg) & $51,9 \pm 13,9$ & $55,8 \pm 14,8$ & $50,9 \pm 13,2$ & 0,014 \\
\hline Estatura $(\mathrm{cm})$ & $139,9 \pm 11,3$ & $142,5 \pm 11,8$ & $139,3 \pm 10,8$ & 0,049 \\
\hline $\mathrm{IMC}\left(\mathrm{kg} / \mathrm{m}^{2}\right)$ & $26,1 \pm 4,1$ & $26,9 \pm 3,9$ & $25,8 \pm 4,0$ & 0,055 \\
\hline IMC z-score & $2,12 \pm 0,33$ & $2,12 \pm 0,32$ & $2,13 \pm 0,33$ & 0,862 \\
\hline Circunferencia de cintura $(\mathrm{cm})$ & $85,9 \pm 10,6$ & $89,3 \pm 11,4$ & $85,1 \pm 9,9$ & 0,004 \\
\hline Presión diastólica (mmHg) & $67,5 \pm 10,9$ & $70,9 \pm 12,9$ & $66,3 \pm 9,9$ & 0,004 \\
\hline Presión sistólica (mmHg) & $104,8 \pm 12,5$ & $109,4 \pm 14,1$ & $103,4 \pm 11,4$ & 0,001 \\
\hline Insulinemia en ayunas ( $\mu \mathrm{U} / \mathrm{mL})$ & $9,75 \pm 9,77$ & $11,99 \pm 7,57$ & $8,95 \pm 10,36$ & 0,034 \\
\hline Glicemia en ayunas (mg/dL) & $88,9 \pm 10,9$ & $91,1 \pm 11,7$ & $88,2 \pm 10,6$ & 0,076 \\
\hline HOMA-IR & $2,16 \pm 2,18$ & $2,73 \pm 1,87$ & $1,95 \pm 2,25$ & 0,014 \\
\hline Colesterol total (mg/dL) & $163,7 \pm 30,3$ & $167,1 \pm 28,1$ & $162,5 \pm 31,1$ & 0,303 \\
\hline Colesterol-LDL (mg/dL) & $95,4 \pm 24,4$ & $96,3 \pm 23,5$ & $95,2 \pm 24,7$ & 0,770 \\
\hline Colesterol-HDL (mg/dL) & $45,9 \pm 9,9$ & $37,5 \pm 6,6$ & $48,8 \pm 9,2$ & $<0,001$ \\
\hline Triglicéridos (mg/dL) & $104,9 \pm 58,1$ & $156,7 \pm 63,5$ & $86,8 \pm 42,9$ & $<0,001$ \\
\hline
\end{tabular}

${ }^{a}$ Niñas y niños con obesidad y síndrome metabólico. ${ }^{b}$ Niñas y niños con obesidad y sin síndrome metabólico. ${ }^{c} p$-value para la comparación de promedios de obesos son SM versus obesos sin SM para la prueba de t de Student.

Tanner y $0,9 \%$ Tanner 5 . Respecto a antecedentes familiares de obesidad, el $27,8 \%$ de los casos tiene un progenitor obeso (IMC $\geq 30 \mathrm{~kg} / \mathrm{m}^{2}$ ), mientras que $12,7 \%$ tiene ambos progenitores obesos. Estos niños y niñas fueron reclutados desde unidades de atención ambulatoria pediátricas del Instituto de Nutrición y Tecnología de los Alimentos (INTA)Universidad de Chile, de la Red de Salud de La Facultad de Medicina de la Pontificia Universidad Católica de Chile y a través de invitaciones abiertas a escuelas del sistema municipal de educación del área sur-oriente de la Región Metropolitana. Todos los progenitores o tutores legales firmaron un documento de consentimiento informado aprobado por los Comités de Ética de Investigación institucionales.

\section{Diagnóstico de síndrome metabólico (SM)}

El SM fue definido por la presencia de al menos 3 de los 5 componentes del fenotipo de Cook: circunferencia de cintura sobre percentil 90 acorde con género y edad, niveles de trigliceridos séricos $>110 \mathrm{mg} / \mathrm{dL}$, colesterol-HDL $<40 \mathrm{mg} / \mathrm{dL}$, glicemia en ayunas $>100 \mathrm{mg} / \mathrm{dL}$, y presión arterial sistólica y/o diastólica $>$ percentil 90 acorde con género, edad y estatura ${ }^{7,43}$.

\section{Genotipificación de SNPs de IL6, IL6R e IL18}

A todos los individuos participantes se les extrajo una muestra de sangre periférica desde donde se obtuvo ADN genómico usando un kit comercial (QIAamp DNA Blood Mini Kit, Qiagen Sciences Inc, Germantwon MD, USA). Todos los polimorfismos fueron genotipificados mediante reacción en cadena de la polimerasa seguida de análisis de fragmentos de restricción (PCR-RFLP) de acuerdo a protocolos previamente descritos ${ }^{21,38,44-46}$.

\section{Análisis estadístico}

Las frecuencias alélicas y genotípicas para todos los polimorfismos fueron estimadas usando proporciones simples. El equilibrio de HardyWeinberg en la distribución de las frecuencias genotípicas fue evaluado a través de una prueba de $\chi^{2}$ de bondad de ajuste. La presencia de asociación entre alelos de estos SNPs y el SM y/o sus compo- 
nentes, se analizó estimado Odds Ratio (OR) con intervalos de confianza de 95\% (IC 95\%). La asociación genotípica se estimó mediante regresión logística, calculando OR con IC 95\%, ajustado por edad y género. Todos los análisis estadísticos se llevaron a cabo con el programa STATA 12.0. Por su parte, la presencia de asociación entre haplotipos conformados por SNPs de IL6, IL18 y el SM y/o sus componentes se llevo a cabo con el programa UNPHASED 3.1.4, que aplica una prueba global de razón de verosimilitud ${ }^{47}$.

\section{Resultados}

De acuerdo a los criterios de $\operatorname{Cook}^{7}$, el número de sujetos de esta muestra diagnosticados con SM son $68(26,3 \%)$ de un total de 259 niñas y niños obesos $^{10}$, en donde las principales variables antropométricas y metabólicas de la muestra se detallan en la Tabla 1. Por su parte, la Tabla 2 muestra la proporción de individuos que exceden los puntos de corte de acuerdo a estos criterios.

La Tabla 3 muestra las frecuencias alélicas de los SNPs de IL6, IL6R e IL18, para los niños y niñas con SM y para aquellos no clasificados en este grupo (no-SM). Al comparar ambos grupos, no existen diferencias significativas en las frecuencias alélicas de ninguna de las siete variantes (Tabla 3). Las frecuencias genotípicas de los siete SNPs analizados en individuos SM y no-SM (Tabla 4) se distribuyeron de acuerdo al equilibrio de HardyWeinberg en ambos grupos (datos no mostrados). Ninguno de los siete SNPs aquí considerados presentó genotipos de riesgo para SM, incluyendo en el modelo las variables edad y género (Tabla 4). Esta ausencia de asociación alélica y genotípica persiste para cada uno de los componentes del SM: circunferencia de cintura $>$ percentil 90, triglicéridos $>110 \mathrm{mg} / \mathrm{dL}$, colesterol-HDL $<40$ $\mathrm{mg} / \mathrm{dL}$, glicemia en ayunas $>100 \mathrm{mg} / \mathrm{dL}$, presión arterial sistólica y/o diastólica $>$ percentil 90 (datos no mostrados).

Entre todos los haplotipos que es posible construir con los SNPs de IL6, por una parte, y con los de IL18 por otra, sólo algunos de este último gen mostraron asociación con ciertos componentes del SM (Tabla 5, orden de los SNPs rs360719-rs187238-rs2043055). Así, el haplotipo TCT $(\mathrm{p}=0,003)$ tendría un efecto protector para el fenotipo circunferencia de cintura $>$ percentil 90. Por su parte el haplotipo CGT $(\mathrm{p}=0,025)$ presenta una frecuencia significativamente mayor

Tabla 2. Componentes del síndrome metabólico en 259 niñas y niños chilenos con obesidad

\begin{tabular}{|lc|}
\hline Componente $^{\mathbf{a}}$ & \% (IC 95\%) \\
\hline $\begin{array}{l}\text { Circunferencia de cintura }>\text { percentil } \\
90\end{array}$ & $97,2(95,2-99,2)$ \\
\hline Hipertensión arterial $^{\mathrm{b}}$ & $25,8(20,3-31,3)$ \\
\hline Glicemia en ayunas $>100 \mathrm{mg} / \mathrm{dL}$ & $11,7(7,7-15,7)$ \\
\hline Colesterol-HDL $<40 \mathrm{mg} / \mathrm{dL}$ & $30,2(24,4-35,9)$ \\
\hline Triglicéridos $>110 \mathrm{mg} / \mathrm{dL}$ & $31,8(26,0-37,6)$ \\
\hline Prevalencia SM & $26,3(20,8-31,7)$ \\
\hline
\end{tabular}

a De acuerdo a los criterios descritos por Cook et al. ${ }^{7}$ bPresión diastólica $>$ percentil 90 o presión sistólica $>$ percentil 90 o ambas.

Tabla 3. Asociación entre alelos de los SNPs de IL6, IL6R e IL18 y el SM en una muestra de 259 niñas y niños con obesidad

\begin{tabular}{|lcccccc|}
\hline Gen & SNP & Alelos $^{\mathbf{a}}$ & $\begin{array}{c}\text { Frecuencia } \\
\mathbf{S M}^{\mathbf{b}} \mathbf{( n = 6 8 )}\end{array}$ & $\begin{array}{c}\text { Frecuencia } \\
\text { No-SMc } \mathbf{( n = 1 9 1 )}\end{array}$ & OR (IC 95\%) & p \\
\hline \multirow{2}{*}{ IL6 } & rs1800797 & G/A & 0,19 & 0,19 & $0,99(0,52-1,82)$ & 0,969 \\
& rs1800796 & G/C & 0,32 & 0,29 & $1,10(0,67-1,81)$ & 0,671 \\
& rs1800795 & G/C & 0,23 & 0,24 & $0,93(0,52-1,62)$ & 0,790 \\
\multirow{2}{*}{ IL6R } & rs8192284 & C/A & 0,37 & 0,37 & $0,98(0,61-1,59)$ & 0,957 \\
\multirow{2}{*}{ IL18 } & rs360719 & T/C & 0,29 & 0,34 & $0,81(0,48-1,37)$ & 0,412 \\
& rs187238 & G/C & 0,34 & 0,32 & $1,11(0,69-1,75)$ & 0,637 \\
& rs2043055 & T/C & 0,56 & 0,49 & $1,31(0,81-2,13)$ & 0,243 \\
\hline
\end{tabular}

${ }^{a}$ Alelo de mayor frecuencia se indica en primer lugar. ${ }^{b}$ Frecuencia del alelo menor en niñas y niños con obesidad y síndrome metabólico. 'Frecuencia del alelo menor en niñas y niños con obesidad y sin síndrome metabólico. 
Polimorfismos de IL6, IL6R e IL18 y síndrome metabólico en obesidad infantil - J. Suazo et al

Tabla 4. Asociación entre genotípos los SNPs de IL6, IL6R e IL18 y el SM en una muestra de 259 niñas y niños con obesidad

\begin{tabular}{|c|c|c|c|c|c|c|c|c|}
\hline \multirow[t]{2}{*}{ Gen } & \multirow[t]{2}{*}{ SNP } & \multirow[t]{2}{*}{ Genotipos } & \multirow[t]{2}{*}{$\begin{array}{l}\text { Frecuencia } \\
\qquad \mathbf{S M}^{\mathrm{a}}\end{array}$} & \multirow[t]{2}{*}{$\begin{array}{c}\text { Frecuencia } \\
\text { No-SM }^{\mathbf{b}}\end{array}$} & \multicolumn{2}{|c|}{ No ajustado } & \multicolumn{2}{|c|}{$\begin{array}{c}\text { Ajustado por edad y } \\
\text { género }\end{array}$} \\
\hline & & & & & OR (IC 95\%) & $\mathbf{p}$ & OR (IC 95\%) & $\mathbf{p}$ \\
\hline \multirow[t]{9}{*}{ IL6 } & rs1800797 & GG & 0,64 & 0,64 & Ref. & & Ref. & \\
\hline & & GA & 0,34 & 0,33 & $1,02(0,51-2,05)$ & 0,947 & $0,92(0,45-1,90)$ & 0,834 \\
\hline & & $A A$ & 0,02 & 0,03 & $0,80(0,08-7,43)$ & 0,844 & $0,47(0,05-4,55)$ & 0,518 \\
\hline & rs1800796 & GG & 0,50 & 0,51 & Ref. & & Ref. & \\
\hline & & GC & 0,37 & 0,40 & $0,93(0,48-1,81)$ & 0,841 & $0,92(0,47-1,82)$ & 0,829 \\
\hline & & $\mathrm{CC}$ & 0,13 & 0,09 & $1,41(0,52-3,84)$ & 0,493 & $1,31(0,47-3,64)$ & 0,608 \\
\hline & rs1800795 & GG & 0,61 & 0,56 & Ref. & & Ref. & \\
\hline & & $\mathrm{GC}$ & 0,33 & 0,40 & $0,77(0,39-1,53)$ & 0,465 & $0,72(0,36-1,44)$ & 0,355 \\
\hline & & CC & 0,06 & 0,04 & $1,37(0,32-5.82)$ & 0,669 & $1,05(0,24-4,72)$ & 0,941 \\
\hline \multirow[t]{3}{*}{$I L 6 R$} & rs8192284 & $C C$ & 0,43 & 0,40 & Ref. & & Ref. & \\
\hline & & CA & 0,40 & 0,46 & $0,78(0,39-1,55)$ & 0,483 & $0,92(0,45-1,88)$ & 0,826 \\
\hline & & AA & 0,17 & 0,14 & $1,11(0,45-2,79)$ & 0,812 & $1,12(0,44-2,87)$ & 0,810 \\
\hline \multirow[t]{9}{*}{$1 L 18$} & & $\mathrm{TT}$ & 0,50 & 0,47 & Ref. & & Ref. & \\
\hline & rs360719 & $\mathrm{TC}$ & 0,42 & 0,39 & $1,00(0,51-1,97)$ & 0,995 & $1,13(0,56-2,29)$ & 0,728 \\
\hline & & $\mathrm{CC}$ & 0,08 & 0,14 & $0,54(0,17-1,74)$ & 0,307 & $0,54(0,16-1,78)$ & 0,314 \\
\hline & & GG & 0,44 & 0,49 & Ref. & & Ref. & \\
\hline & rs187238 & GC & 0,43 & 0,39 & $1,07(0,55-2,08)$ & 0,834 & $1,31(0,65-2,63)$ & 0,437 \\
\hline & & CC & 0,13 & 0,12 & $0,93(0,33-2,61)$ & 0,896 & $0,98(0,34-2,82)$ & 0,971 \\
\hline & rs2043055 & $\mathrm{TT}$ & 0,18 & 0,26 & Ref. & & Ref. & \\
\hline & & $\mathrm{TC}$ & 0,51 & 0,49 & $1,49(0,65-3,42)$ & 0,341 & $1,67(0,69-4,02)$ & 0,252 \\
\hline & & $\mathrm{CC}$ & 0,31 & 0,25 & $2,17(0,86-5,43)$ & 0,098 & $1,93(0,73-5,05)$ & 0,183 \\
\hline
\end{tabular}

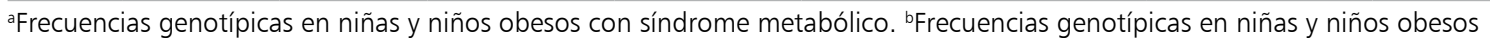
sin síndrome metabólico.

en individuos con niveles de triglicéridos $\leq 110$ $\mathrm{mg} / \mathrm{dL}$. Por último, la combinación alélica TCT $(\mathrm{p}=0,016)$ incrementaría el riesgo de presentar niveles plasmáticos de $\mathrm{HDL}<40 \mathrm{mg} / \mathrm{dL}$ (Tabla 5).

\section{Discusión}

Estudios previos indican que, en conjunto, los polimorfismos genéticos de IL6 IL6R analizados en este estudio, regularían los niveles de IL6 y su función ${ }^{29-32}$. Adicionalmente, la relación antes descrita entre IL6, inflamación y alteraciones metabólicas, permitiría postular la presencia de asociación entre variantes de estos genes y SM y/o sus componentes. Sin embargo, en nuestra muestra de niñas y niños obesos chilenos, no se detectó asociación entre alelos, genotipos ni haplotípos de IL6 e IL6R tanto con SM como para ninguno de sus componentes, situación similar a la de otros estudios ${ }^{48-50}$.
Nuestros hallazgos podrían estar influenciados por factores como la edad. Las alteraciones del sistema inmune en el envejecimiento, se manifiestan como incremento de los niveles circulantes de mediadores de la inflamación como las interleuquinas, agravando así patologías asociadas a la edad $^{51}$. La mayoría de los reportes que relacionan niveles de IL6 y/o sus variantes genéticas con componentes del SM, se han llevado a cabo en adultos $^{21,28,39,48,52}$. En este contexto, linfocitos B inmortalizados de adultos mayores (89 años promedio) portadores del alelo C de rs 1800795 (promotor de IL6), producen significativamente más IL6 que linfocitos, también provenientes de portadores de este alelo, pero con 35 años de edad promedio ${ }^{53}$. Sin embargo, algunos de los polimorfismos aquí analizados muestran asociación con componentes del SM en adolescentes. Es así como la variante IL6R rs2228145 ha sido asociada con incremento de la circunferencia abdominal y triglicéridos y 
bajos niveles de HDL en niñas adolescentes de Taiwan (edad promedio 13,1 años; IMC promedio $\left.20,5 \mathrm{~kg} / \mathrm{m}^{2}\right)^{40}$. En adolescentes españoles (edad promedio 14,5 años; IMC Z-score promedio 1,05), el polimorfismo rs 1800795 de IL6, aunque no se asocia directamente con ningún componente del $\mathrm{SM}$, tiene influencia sobre la relación porcentaje de grasa corporal-glicemia en ayunas ${ }^{54}$. Esto refleja la presencia de otros factores como el género y el origen étnico, que se ha demostrado que son las principales variables que influencian los niveles de IL $6^{55}$. En dicho reporte, las mujeres presentaron mayores niveles de esta interleuquina que los varones, diferencia que los autores atribuyen al efecto de las hormonas sexuales.

Respecto al origen étnico, los afroamericanos muestran mayores niveles circulantes de IL6 que los caucásicos ${ }^{55}$. En este contexto, y de acuerdo al origen propuesto de la población chilena contemporánea ${ }^{56}$, las frecuencias del alelo menor de los polimorfismos de IL6 en nuestra muestra se acercan más a los valores descritos para amerindios que para caucásicos $(0,22,0,29$ y 0,18 para rs1800795, rs1800796 rs1800797, respectivamente). En cambio, la frecuencia de rs 2228145 en IL6R, es muy similar a la de caucásicos (http:// hapmap.ncbi.nlm.nih.gov/). Existe la posibilidad que estas frecuencias no sean suficientes para detectar riesgos genotípicos bajos (como los que habitualmente se describen en enfermedades complejas) con el número de casos de SM considerados en este estudio $(n=68$, en una población de 259 individuos).

Para las variantes polimórficas del gen IL18, en nuestra muestra no se observaron alelos ni genotipos de riesgo para SM ni para ninguno de los fenotipos que lo componen. Sin embargo, se detectan algunos haplotipos, construidos con los alelos de estos SNPs, que muestran asociación con ciertos componentes del SM (Tabla 5). Así, el haplotipo TCT tendría un rol protector ante valores alterados de circunferencia de cintura ( $>$ percentil 90), resultado que parece ser el reflejo del reducido número de individuos que presentan valores normales para este fenotipo (11 de 259) y por ende, de las frecuencias haplotípicas observadas. El haplotipo CGT mostró tener un rol protector para los niveles elevados de triglicéridos al presentar una frecuencia significativamente mayor en sujetos con valores $\leq 110 \mathrm{mg} / \mathrm{dL}$. Por su parte el haplotipo TCT se asocia con niveles reducidos de HDL $(<40 \mathrm{mg} / \mathrm{dL})$. Las diferencias entre los análisis alelico/genotípico y haplotipico pueden explicarse por el mayor poder del uso de haplotipos para detectar asociaciones genotipo-fenotipo, versus el uso de marcadores individuales ${ }^{57-58}$. En cualquier caso, los haplotipos permiten capturar una mayor variabilidad genética (por desequilibrio de ligamiento) en relación al uso de variantes aisladas ${ }^{59}$.

Los niveles circulantes de IL18 parecen ser influenciados por las variantes funcionales en la región promotora de IL18 consideradas en este trabajo. Es así como los portadores del alelo $\mathrm{C}$ de rs360719 muestran un incremento en la expresión del RNA mensajero de este gen versus el alelo $\mathrm{T}^{36}$. En el caso de rs187238, el alelo G se correlaciona con una mayor actividad transcripcional del promotor $^{33}$. Por su parte, el alelo C del SNP intrónico rs204355 se asocia con mayores niveles circulantes de esta interleuquina ${ }^{35}$. Dado que se ha reportado que a medida que aumentan los niveles de IL18 se incrementan los triglicéridos y disminuye el colesterol-HDL ${ }^{60}$, nuestros resultados parecen paradójicos. El haplotipo asociado a niveles normales de triglicéridos porta alelos correlacionados con

Tabla 5. Haplotipos conformados por los SNPs de IL18 que muestran asociación con componentes del SM en una muestra de 259 niñas y niños con obesidad

\begin{tabular}{|c|c|c|c|}
\hline Haplotipo ${ }^{a}$ & & encias & $\mathbf{p}$ \\
\hline $\begin{array}{l}\text { Circunferencia de cintura } \\
\text { T-C-T }\end{array}$ & $\begin{array}{l}>\text { Percentil } 90(n=248) \\
0,02\end{array}$ & $\begin{array}{l}\leq \text { Percentil } 90(n=11) \\
0,18\end{array}$ & 0,003 \\
\hline $\begin{array}{c}\text { Triglicéridos } \\
\text { C-G-T }\end{array}$ & $\begin{array}{l}>110 \mathrm{mg} / \mathrm{dL}(\mathrm{n}=82) \\
0,01\end{array}$ & $\begin{array}{l}\leq 110 \mathrm{mg} / \mathrm{dL}(\mathrm{n}=177) \\
0,07\end{array}$ & 0,025 \\
\hline $\begin{array}{l}\mathrm{CHDL} \\
\mathrm{T}-\mathrm{C}-\mathrm{T}\end{array}$ & $\begin{array}{l}<40 \mathrm{mg} / \mathrm{dL}(\mathrm{n}=78) \\
0,06\end{array}$ & $\begin{array}{l}\geq 40 \mathrm{mg} / \mathrm{dL}(\mathrm{n}=181) \\
0,01\end{array}$ & 0,016 \\
\hline
\end{tabular}

${ }^{a}$ Orden de los SNPs en el haplotipo: rs360719-rs187238-rs2043055. 
Polimorfismos de IL6, IL6R e IL18 y síndrome metabólico en obesidad infantil - J. Suazo et al

niveles mayores de esta interleuquina (rs360719-C y rs187238-G). En el caso de los niveles de HDL, se esperaría que los portadores del haplotipo asociado a este fenotipo (TCT) tuviesen menores niveles de IL18 y, por lo tanto, mayores valores de HDL. Sin embargo, todas estas inferencias se basan en los efectos individuales descritos para cada alelo de estas tres variantes y en adultos, por lo que no necesariamente son extrapolables a nuestra muestra. Además, nuestros resultados pueden verse influenciados por las relativamente bajas frecuencias de los haplotipos CGT y TCT en nuestra población (Tabla 5). Esto devela algunas de las debilidades de nuestro estudio, por lo que se hacen necesarios trabajos independientes con tamaños muestrales mayores, que consideren el efecto de estos haplotipos sobre los niveles circulantes de IL18 (u otros marcadores de inflamación) en la infancia.

En síntesis, en nuestra población las variantes de los genes IL6 e IL6R no se asocian con un incremento del riesgo de presentar SM ni con ninguno de los fenotipos que lo componen. Estos resultados podrían ser explicados por el efecto de una serie de factores tales como la edad, el género, el origen étnico o la magnitud de la asociación esperada. Sin embargo, ciertos haplotipos, de baja frecuencia, conformados por los polimorfismos de IL18 se asocian con niveles normales de triglicéridos $(\leq 110 \mathrm{mg} / \mathrm{dL})$ y niveles anormalmente bajos de HDL $(<40 \mathrm{mg} / \mathrm{dL})$. Consideramos necesario estudios independientes que repliquen nuestros hallazgos.

Agradecimientos: Los autores desean agradecer la colaboración de las niñas y niños participantes de este estudio y de sus familias, sin la cual este estudio no habría sido posible. Este trabajo fue financiado por el proyecto del Fondo Nacional para el Desarrollo de la Ciencia y la Tecnología (FONDECYT) número 1090388.

\section{Referencias}

1. Kahn R. Metabolic syndrome: is it a syndrome? Does it matter? Circulation 2007; 115 (13): 1806-10.

2. Reaven GM. Insulin resistance, the insulin resistance syndrome, and cardiovascular disease. Panminerva Med 2005; 47 (4): 201-10.

3. Ogden CL, Carroll MD, Kit BK, Flegal KM. Prevalence of obesity in the United States, 2009-2010. NCHS Data
Brief 2012; 82 (1): 1-8.

4. Albala C, Vio F, Kain J, Uauy R. Nutrition transition in Chile: determinants and consequences. Public Health Nutr 2002; 5 (1A): 123-8.

5. Kain J, Uauy R, Vio F, Albala C. Trends in overweight and obesity prevalence in Chilean children: comparison of three definitions. Eur J Clin Nutr 2002; 56 (3): 200-4.

6. Sanjurjo-Crespo P, Prieto Perera JA, Andrade Lodeiro F, Aldámiz-Echevarría Azuara L. Metabolic syndrome in childhood. Public Health Nutrition 2007; 10 (10A): 1121-5.

7. Cook S, Weitzman M, Auinger P, Nguyen M, Dietz WH. Prevalence of a metabolic syndrome phenotype in adolescents: findings from the third National Health and Nutrition Examination Survey, 1988-1994. Arch Pediatr Adolesc Med 2003; 157 (8): 821-7.

8. Johnson WD, Kroon JJ, Greenway FL, Bouchard C, Ryan D, Katzmarzyk PT. Prevalence of risk factors for metabolic syndrome in adolescents: National Health and Nutrition Examination Survey (NHANES), 2001-2006. Arch Pediatr Adolesc Med 2009; 163 (4): 371-7.

9. Guijarro de Armas MA, Monereo Megías S, Merino Viveros M, Iglesias Bolaños P, Vega Piñero B. Prevalence of metabolic syndrome in a population of obese children and adolescents. Endocrinol Nutr 2012; 59 (3): 155-9.

10. Suazo J, Hodgson MI, Obregón AM, Valladares M, Weisstaub G, Amador P, et al. Prevalence of metabolic syndrome in obese Chilean children and association with gene variants of the leptin-melanocortin system. J Pediatr Endocrinol Metab 2013. doi: 10.1515/jpem2013-0084.

11. Park HS, Park JY, Cho SI. Familial aggregation of the metabolic syndrome in Korean families with adolescents. Atherosclerosis 2006; 186 (1): 215-21.

12. Tang W, Hong Y, Province MA, Rich SS, Hopkins PN, Arnett DK. Familial clustering for features of the metabolic syndrome: the National Heart, Lung, and Blood Institute (NHLBI) Family Heart Study. Diabetes Care 2006; 29 (3): 631-6.

13. Sookoian S, Pirola CJ. Genetics of the cardiometabolic syndrome: new insights and therapeutic implications. Ther Adv Cardiovasc Dis 2007; 1 (1): 37-47.

14. Sookoian S, Pirola CJ. Metabolic syndrome: from the genetics to the pathophysiology. Curr Hypertens Rep 2011; 13 (2): 149-57.

15. Dinarello CA. Interleukin 1 and interleukin 18 as mediators of inflammation and the aging process. Am J Clin Nutr 2006; 83 (2): 447S-55S.

16. Tam CS, Clément K, Baur LA, Tordjman J. Obesity and low-grade inflammation: a paediatric perspective. Obes Rev 2010; 11 (2): 118-26. 
17. Zorrilla EP, Sánchez-Alavez M, Sugama S, Brennan M, Fernández R, Bartfai T, et al. Interleukin-18 controls energy homeostasis by suppressing appetite and feed efficiency. Proc Natl Acad Sci USA 2007; 104 (26): 11097-102.

18. Pradhan AD, Manson JE, Rifai N, Buring JE, Ridker PM. C-reactive protein, interleukin 6 , and risk of developing type 2 diabetes mellitus. JAMA 2001; 286 (3): 327-34.

19. Vozarova B, Weyer C, Hanson K, Tataranni PA, Bogardus C, Pratley RE. Circulating interleukin-6 in relation to adiposity, insulin action, and insulin secretion. Obes Res 2001; 9 (7): 414-7.

20. Esposito K, Pontillo A, Ciotola M, Di Palo C, Grella E, Nicoletti G, et al. Weight Loss Reduces Interleukin-18 Levels in Obese Women. J Clin Endocrinol Metab 2002; 87 (8): 3864-66.

21. Bustamante M, Nogués X, Mellibovsky L, Agueda L, Jurado S, Cáceres E, et al. Polymorphisms in the interleukin-6 receptor gene are associated with bone mineral density and body mass index in Spanish postmenopausal women. Eur J Endocrinol 2007; 157 (5): 677-84.

22. Zirlik A, Abdullah SM, Gerdes N, MacFarlane L, Schönbeck $U$, Khera $A$, et al. Interleukin-18, the metabolic syndrome, and subclinical atherosclerosis: results from the Dallas Heart Study. Arterioscler Thromb Vasc Biol 2007; 27 (9): 2043-9.

23. Smart MC, Dedoussis G, Yiannakouris N, Grisoni ML, Dror GK, Yannakoulia M, et al. Genetic variation within IL18 is associated with insulin levels, insulin resistance and postprandial measures. Nutr Metab Cardiovasc Dis 2011; 21 (7): 476-84.

24. Brull DJ, Montgomery HE, Sanders J, Dhamrait S, Luong L, Rumley A, et al. Interleukin-6 gene $-174 \mathrm{~g}>\mathrm{c}$ and $-572 \mathrm{~g}>\mathrm{c}$ promoter polymorphisms are strong predictors of plasma interleukin-6 levels after coronary artery bypass surgery. Arterioscler Thromb Vasc Biol 2001; 21 (9): 1458-63.

25. Rafiq S, Frayling TM, Murray A, Hurst A, Stevens K, Weedon $\mathrm{MN}$, et al. A common variant of the interleukin 6 receptor (IL-6r) gene increases IL-6r and IL-6 levels, without other inflammatory effects. Genes Immun 2007; 8 (7): 552-9.

26. Reich D, Patterson N, Ramesh V, De Jager PL, McDonald GJ, Tandon A, et al. Admixture mapping of an allele affecting interleukin 6 soluble receptor and interleukin 6 levels. Am J Hum Genet 2007; 80 (4): 716-26.

27. Hawkins GA, Robinson MB, Hastie AT, Li X, Li H, Moore WC, et al. The IL6R variation Asp(358)Ala is a potential modifier of lung function in subjects with asthma. J Allergy Clin Immunol 2012; 130 (2): 510-5.

28. Qi L, Zhang C, van Dam RM, Hu FB. 2007. Interleu- kin-6 genetic variability and adiposity: associations in two prospective cohorts and systematic review in 26,944 individuals. J Clin Endocrinol Metab 92: 3618-25.

29. Terry CF, Loukaci V, Green FR. Cooperative influence of genetic polymorphisms on interleukin 6 transcriptional regulation. J Biol Chem 2000; 275 (24): 18138-44.

30. Qi L, Rifai N, Hu FB. Interleukin-6 receptor gene variations, plasma interleukin- 6 levels, and type 2 diabetes in U.S. Women. Diabetes 2007; 56 (12): 3075-81.

31. Jones SA, Horiuchi S, Topley N, Yamamoto N, Fuller GM. The soluble interleukin 6 receptor: mechanisms of production and implications in disease. FASEB J 2001; 15 (1): 43-58.

32. Jones SA, Novick D, Horiuchi S, Yamamoto N, Szalai AJ, Fuller GM. C-reactive protein: a physiological activator of interleukin 6 receptor shedding. J Exp Med 1999; 189 (3): 599-604.

33. Liang XH, Cheung W, Heng CK, Wang DY. Reduced transcriptional activity in individuals with IL-18 gene variants detected from functional but not association study. Biochem Biophys Res Commun 2005; 338 (2): 736-41.

34. Arimitsu J, Hirano T, Higa S, Kawai M, Naka T, Ogata A, et al. IL-18 gene polymorphisms affect IL-18 production capability by monocytes. Biochem Biophys Res Commun 2006; 342 (4): 1413-6.

35. Thompson SR, McCaskie PA, Beilby JP, Hung J, Jennens $\mathrm{M}$, et al. IL18 haplotypes are associated with serum IL-18 concentrations in a population-based study and a cohort of individuals with premature coronary heart disease. Clin Chem 2007; 53 (12): 2078-85.

36. Sánchez E, Palomino-Morales RJ, Ortego-Centeno N, Jiménez-Alonso J, González-Gay MA, López-Nevot MA, et al. Identification of a new putative functional IL18 gene variant through an association study in systemic lupus erythematosus. Hum Mol Genet 2009; 18 (19): 3739-48.

37. Evans J, Collins M, Jennings C, van der Merwe L, Söderström I, Olsson T, et al. The association of interleukin-18 genotype and serum levels with metabolic risk factors for cardiovascular disease. Eur J Endocrinol 2007; 157 (5): 633-40.

38. Thompson SR, Sanders J, Stephens JW, Miller GJ, Humphries SE. A common interleukin 18 haplotype is associated with higher body mass index in subjects with diabetes and coronary heart disease. Metabolism 2007; 56 (5): 662-9.

39. Jiang CQ, Lam TH, Liu B, Lin JM, Yue XJ, Jin YL, et al. Interleukin-6 receptor gene polymorphism modulates interleukin-6 levels and the metabolic syndrome: GBCSCVD. Obesity (Silver Spring) 2010; 18 (10): 1969-74. 
40. Hsieh CH, Hung YJ, Wu LI, He CT, Lee CH, Hsiao FC, et al. Interleukin-6 receptor gene $48892 \mathrm{~A} / \mathrm{C}$ polymorphism is associated with metabolic syndrome in female Taiwanese adolescents. Genet Test Mol Biomarkers 2012; 16 (12): 1376-81.

41. Pérez-Bravo F, Oyarzún AM, Soto F, López P, Eyzaguirre F, Codner E. Polymorphisms in the interleukin-6 receptor gene (Asp358Ala) and body mass index in Chilean women with type 1 diabetes. Endocr Res 2012; 37 (4): 197-202.

42. Centers for Disease Control and Prevention/National Center for Health Statistics (2000) CDC growth charts: United States. Available at: http://www.cdc.gov/growthcharts/.

43. National High Blood Pressure Education Program Working Group on High Blood Pressure in Children and Adolescents. The fourth report on the diagnosis, evaluation, and treatment of high blood pressure in children and adolescents. Pediatrics 2004; 114 (2 Suppl 4th Report): 555-76.

44. Pawlik A, Kurzawski M, Drozdzik M, Dziedziejko V, Safranow K, Herczynska M. Interleukin-18 gene (IL18) promoter polymorphisms in patients with rheumatoid arthritis. Scand J Rheumatol 2009; 38 (3): 159-65.

45. Grzegorzewska AE, Wobszal PM, Mostowska A, Jagodziński PP. Antibodies to hepatitis B virus surface antigen and interleukin 12 and interleukin 18 gene polymorphisms in hemodialysis patients. BMC Nephrol 2012; $13: 75$.

46. Rasmussen L, Delabio R, Horiguchi L, Mizumoto I, Terazaki CR, Mazzotti D, et al. Association Between Interleukin 6 Gene Haplotype and Alzheimer's Disease: A Brazilian Case-Control Study. J Alzheimers Dis 2013; mayo 10 [Epub ahead of print].

47. Dudbridge F. Likelihood-based association analysis for nuclear families and unrelated subjects with missing genotype data. Hum Hered 2008; 66 (2): 87-98.

48. Hamid YH, Rose CS, Urhammer SA, Glümer C, Nolsøe $\mathrm{R}$, Kristiansen OP, et al. Variations of the interleukin-6 promoter are associated with features of the metabolic syndrome in Caucasian Danes. Diabetologia 2005; 48 (2): 251-60.

49. Phillips CM, Goumidi L, Bertrais S, Ferguson JF, Field MR, Kelly ED, et al. Additive effect of polymorphisms in the IL-6, LTA, and TNF-\{alpha\} genes and plasma fatty acid level modulate risk for the metabolic syndrome and its components. J Clin Endocrinol Metab 2010; 95 (3): 1386-94.
50. Cheung BM, Ong KL, Tso AW, Leung RY, Cherny SS, Sham PC, et al. Relationship of plasma interleukin-6 and its genetic variants with hypertension in Hong Kong Chinese. Am J Hypertens 2011; 24 (12): 1331-7.

51. Krabbe KS, Pedersen M, Bruunsgaard H. Inflammatory mediators in the elderly. Exp Gerontol 2004; 39 (5): 687 99.

52. Grallert H, Huth C, Kolz M, Meisinger C, Herder C, Strassburger K, et al. IL-6 promoter polymorphisms and quantitative traits related to the metabolic syndrome in KORA S4. Exp Gerontol 2006; 41 (8): 737-45.

53. Olivieri F, Bonafe M, Giovagnetti S, Stecconi R, Cardelli $\mathrm{M}$, Cavallone $\mathrm{L}$, et al. In vitro IL-6 production by EBVimmortalized B lymphocytes from young and elderly people genotyped for $-174 \mathrm{C} / \mathrm{G}$ polymorphism in IL-6 gene: a model to study the genetic basis of inflammaging. Mech Ageing Dev 2003; 124 (4): 549-53.

54. Moleres A, Rendo-Urteaga T, Azcona C, Martínez JA, Gómez-Martínez S, Ruiz JR, et al. Il6 gene promoter polymorphism $(-174 \mathrm{G} / \mathrm{C})$ influences the association between fat mass and cardiovascular risk factors. J Physiol Biochem 2009; 65 (4): 405-13.

55. Chapman BP, Khan A, Harper M, Stockman D, Fiscella $\mathrm{K}$, Walton J, et al. Gender, race/ethnicity, personality, and interleukin- 6 in urban primary care patients. Brain Behav Immun 2009; 23 (5): 636-42.

56. Rothhammer F, Lasserre E, Blanco R, Covarrubias E, Dixon M. Microevolution in human Chilean populations. IV. Shovel shape, mesial-palatal version and other dental traits in Pewenche Indians. Z Morphol Anthropol 1968; 60 (2): 162-9.

57. Escamilla MA, McInnes LA, Spesny M, Reus VI, Service SK, Shimayoshi N, et al. Assessing the feasibility of linkage disequilibrium methods for mapping complex traits: an initial screen for bipolar disorder loci on chromosome 18. Am J Hum Genet 1999; 64 (6): 1670-8.

58. Martin ER, Lai EH, Gilbert JR, Rogala AR, Afshari AJ, Riley J, et al. SNPing away at complex diseases: analysis of single-nucleotide polymorphisms around APOE in Alzheimer disease. Am J Hum Genet 2000; 67 (2): 38394.

59. Akey J, Jin L, Xiong M. Haplotypes vs single marker linkage disequilibrium tests: what do we gain? Eur J Hum Genet 2001; 9 (4): 291-300.

60. Jefferis BJ, Papacosta O, Owen CG, Wannamethee SG, Humphries SE, Woodward M, et al. Interleukin 18 and coronary heart disease: prospective study and systematic review. Atherosclerosis 2011; 217 (1): 227-33. 